\title{
Psoriatic Arthritis (1st Edition)
}

Dafna Gladman, Cheryl F. Rosen, and Vinod Chandran. Oxford: Oxford University Press, 2014, 112 pages, \$25 US

When asked to review Psoriatic Arthritis, I was expecting a hefty tome, but the book arrived in a small envelope, an indication of the size of this "pocket guide". This paperback book measures $13 \mathrm{~cm}$ by $19 \mathrm{~cm}$, is 94 pages long, and has a few black and white figures. So much for the basics - what about the substance? As would be expected from such distinguished authors, the facts are succinct and relevant. The chapters follow the usual format from classification criteria to prognosis and are up to date at the time of writing. As with all hard-copy books, the pace of life leaves them behind and they rapidly become out of date. Nevertheless, for people studying for examinations and for internists, this is a handy book that can be pulled out of the pocket and consulted - not quite as contemporary as the smart phone, but much more pleasant to use.

PHILIP S. HELLIWELL, MA, DM, PhD, FRCP, University of Leeds, Section of Musculoskeletal Disease, 2nd Floor, Chapel Allerton Hospital, Harehills Lane, Leeds, LS7 4SA, UK. E-mail: p.helliwell@leeds.ac.uk

J Rheumatol 2014;41:11; doi:10.3899/jrheum.140852 